\title{
Repercusiones económicas y sociales de los festivales culturales: el caso del Festival Internacional de Cine de Valdivia
}

\author{
María Devesa. Universidad de Valladolid, Valladolid, España. \\ Andrea Báez. Universidad Austral de Chile, Valdivia, Chile. \\ Víctor Figueroa. Universidad Austral de Chile, Valdivia, Chile. \\ Luis C. Herrero. Universidad de Valladolid, Valladolid, España.
}

RESUMEN | Hoy, y debido a sus repercusiones en los territorios, la cultura y los festivales culturales forman parte de las estrategias de desarrollo local y regional, y de las actuaciones de regeneración urbana. Tales repercusiones pueden ser de carácter social, cultural, turístico o económico, ya que la cultura genera impactos en términos de producción, rentas y empleos. En este marco, el objetivo de este trabajo es analizar la contribución económica del Festival Internacional de Cine de Valdivia a la ciudad y región donde se celebra. Para ello se utiliza la metodología de los Estudios de Impacto Económico, basada en la definición y medición de tres tipos de efectos (directos, indirectos e inducidos). Los resultados muestran que el festival genera una repercusión significativa sobre la economía de la zona, beneficiando especialmente al sector turístico de la ciudad, y a los servicios a empresas. El trabajo pone también de manifiesto la importancia que los espectadores dan a las repercusiones culturales del festival, que constituyen, en realidad, la esencia del mismo.

PALABRAS CLAVE | Capital cultural, competitividad urbana, desarrollo regional y local

ABSTRACT | Today, culture and cultural festivals are part of the strategies of local and regional development and urban regeneration actions as a result of the impact that these goods can generate on the territories. These repercussions could be social, cultural, touristic or economic, since cultural goods and cultural events generate impacts in terms of production, income and jobs. The aim of this paper is to analyze the economic contribution of the Valdivia International Film Festival to the city and region where it is held. The Economic Impact Study methodology is used for this purpose, based on the definition and valuation of three types of effects (direct, indirect and induced). The results show that the festival generates a significant impact on the economy of the area, benefiting especially the tourism sector of the city and business services. The work also highlights the importance attached by the attendees to the cultural impacts of the festival, which are, in fact, the essence of the event.

KEY WORDS | Cultural capital, urban competitiveness, regional and local development

Recibido el 28 de julio de 2010, aprobado el 15 de agosto de 2011

E-mail: María Devesa, mdevesa@eco.uva.es | Andrea Báez, abaez@uach.cl | Víctor Figueroa, vfiguero@uach.cl | Luis C. Herrero, herrero@emp. uva.es 


\section{Introducción}

En los últimos años se ha producido un uso creciente del factor cultural como determinante de las políticas de desarrollo local y regional y de las estrategias de regeneración urbana (Bianchini \& Parkinson, 1994; Lim, 1993; Scott, 2000). En efecto, parece confiarse en las actividades culturales como fuente de riqueza y empleo, eminentemente por la atracción de flujos de gastos y rentas ligados al turismo cultural, pero también mediante la transformación y desarrollo del tejido productivo que, en conjunto, procuran nuevas ventajas comparativas para las ciudades y las regiones.

A su vez, y derivado de este fenómeno, la cultura se está convirtiendo en un factor de ordenación urbana, no solo por lo que implica la dotación de nuevas infraestructuras culturales y la regeneración de espacios urbanos, sino también por la diversificación de la oferta cultural y los reclamos turísticos que, en suma, tratan de configurar una imagen más atractiva y representativa de la calidad de vida, capaz de competir con otros enclaves o territorios mediante esa estrategia de diferenciación. De este modo, las ciudades y las regiones compiten entre sí en la satisfacción de las necesidades de los diferentes grupos de usuarios — visitantes, residentes, inversores y empresas - y la cultura refuerza, en ese sentido, la competitividad de la zona (Evans, 2001; Gibson \& Stevenson, 2004; Richards \& Wilson, 2004). En este inicio del siglo XXI, hay una marcada convergencia entre la esfera cultural y la económica, lo que supone una de las características distintivas de los procesos contemporáneos de urbanización, donde la cultura no se entiende tanto como una dotación heredada, sino como un potencial creativo capaz de proyectar nuevas ventajas comparativas y también nuevos factores de diferenciación urbana (Scott, 2000). Hoy las ciudades compiten en el desarrollo cultural y creativo para atraer una nueva clase creativa (Florida, 2002), y lo hacen a partir de la producción cultural de base tecnológica, que ayuda a desarrollar la imagen y los símbolos que son vitales para el éxito competitivo de la nueva economía (Richards \& Palmer, 2010).

En este contexto hay que entender el crecimiento de la oferta de actividades culturales de las ciudades y regiones, paralelo a un ascenso notable en el interés por la cultura y en el consumo cultural (Frey, 1994). Ejemplos de esta tendencia son el esfuerzo reciente en la restauración y puesta en valor de numerosos legados patrimoniales; el boom museístico de los últimos años; o la mejora de las infraestructuras de las artes escénicas, como auditorios, teatros y palacios de festivales y congresos. Asimismo destaca el aumento del número de eventos y festivales culturales, en un fenómeno que algunos autores han denominado como de festivalización de las ciudades (Richards, 2007).

Los festivales culturales constituyen uno de los prototipos más representativos del patrimonio cultural y, a la vez, se han convertido en uno de los fenómenos más dinámicos del panorama cultural actual. Su número ha crecido sustancialmente en los últimos años, de manera que varias ciudades en los distintos continentes tienen uno o varios festivales dedicados a alguna manifestación artística. En la actualidad, los festivales no solo cumplen su papel tradicional como institución que permite 
presentar, difundir o preservar la cultura de una sociedad, sino que destacan también por su capacidad para generar riqueza en las ciudades donde se celebran, por su contribución a la diversificación del producto turístico o a la mejora de la imagen del lugar. Los festivales tienen, en definitiva, una dimensión cultural, económica y turística que no es nueva en esencia, pero sí en cuanto a su expansión en los últimos años, lo que ha suscitado un interés creciente de la comunidad científica.

Los festivales culturales tienen una relación importante con el lugar donde se celebran, ya que, por una parte, vienen condicionados por el territorio, su historia y sus gentes; $y$, por otra, generan repercusiones e impactos en los lugares donde se celebran y organizan. Esos impactos han sido analizados tanto desde un punto de vista teórico como práctico, y desde distintas disciplinas académicas (economía, geografía, sociología, marketing o turismo), por lo que existen diferentes maneras de abordar su estudio y clasificación. Así, algunos autores como Moscardo (2008) hablan de impactos económicos, físicos, socioculturales, psicológicos, turísticos y de desarrollo regional de la comunidad. Otros, como Quinn (2005), analizan el efecto de los festivales como creadores de imagen o como atractivos turísticos, el papel de los eventos en la creación de un sentido de comunidad o su desempeño en el binomio globalización y diversidad local. También Crespi-Vallbona y Richards (2007) analizan los efectos de los festivales y eventos desde una doble perspectiva: los festivales en el desarrollo urbano y artístico (festivales como catalizadores de renovación urbana, atracción de turismo, mejora de la imagen y creadores de empleo) y los festivales en ciudades habitables (el papel de los festivales en la mejora de los valores cívicos de la sociedad, desarrollo de la identidad y sentido del lugar).

En términos generales, podemos distinguir distintos tipos de impactos o repercusiones de los festivales y eventos culturales en el lugar donde se celebran; son impactos cuya delimitación es imprecisa, ya que están interrelacionados entre sí, pero, pese a ello, es posible mencionar los cinco siguientes:

a) Repercusiones económicas. Los efectos económicos que los festivales culturales pueden generar sobre el desarrollo económico local y regional se pueden dividir en dos grandes grupos. Por una parte, los efectos a corto plazo, relacionados con el poder de atracción y creación de gasto de estos eventos. Los festivales atraen a espectadores locales y visitantes que gastan su renta en las actividades culturales, así como en otros bienes y servicios relacionados, lo que a su vez provoca efectos de arrastre sobre el resto de la economía. Estos impactos pueden ser medidos por metodologías de evaluación económica, como los Estudios de Impacto Económico (vid. in extenso Devesa Fernández, 2006). Por otra parte, están los efectos económicos a largo plazo, que hacen referencia a las modificaciones de la estructura productiva, urbana y social del territorio, y están relacionados a su vez con los efectos culturales, sociales o físicos. Podemos distinguir aquí el rescate de edificios y la ordenación urbana; la capacidad para atraer residentes, empresas e inversiones; la mejora del nivel educativo y fomento de la creatividad; la mejora de la posición competitiva del territorio; y 
los avances sociales en términos de cohesión social, bienestar de los ciudadanos o sentido de identidad (vid. in extenso Herrero Prieto, 2011).

b) Repercusiones turisticas. Los festivales culturales constituyen en la actualidad un recurso importante para ciudades y regiones (Weiler \& Hall, 1992), así como uno de los atractivos más destacados dentro del turismo (Chacko \& Schaffer, 1993; Crompton \& McKay, 1997; Grant \& Paliwoda, 1998). La celebración de un festival puede ser un factor decisivo en la elección de un destino, una razón de peso en la repetición de la visita o un elemento que mejore la satisfacción de los visitantes, lo que permite desarrollar el perfil turístico y la imagen cultural del lugar (Getz, 1997). Evidentemente, el poder de atracción variará de un caso a otro, pero su potencial colectivo como recurso turístico parece hoy ilimitado. Ello genera, a su vez, un impacto económico en términos de producción, rentas y empleo.

c) Repercusiones culturales. Un evento cultural, por definición, tiene su foco puesto en la cultura, aunque pueda contener otros elementos. En ese sentido, los festivales son un lugar donde ver y participar en manifestaciones artísticas que no se pueden ver el resto del año. Proporcionan oportunidades culturales para las audiencias - ya sean locales o foráneas- que muchas veces son innovadoras, vanguardistas y novedosas, y que complementan, por tanto, las infraestructuras culturales fijas. Son bienes culturales complejos donde las actuaciones no están aisladas, sino que forman parte de un proceso con un objetivo determinado. Son centros de transferencia cultural, donde se consume cultura (los espectadores), se reproduce cultura (obras de teatro, por ejemplo) e incluso se produce cultura (un ambiente determinado, debate o creatividad, pero también productos concretos, como por ejemplo las orquestas propias de algunos festivales). Funcionan, en ocasiones, como faros para la creación artística, ya que pueden asumir riesgos artísticos mayores que las infraestructuras culturales fijas (Frey \& Busenhart, 1996). Ello permite ofrecer una cultura alternativa, diferente y a veces innovadora que aumenta la oferta cultural de las ciudades y regiones.

d) Repercusiones sociales. Los festivales culturales pueden contribuir al desarrollo social, tanto desde un punto de vista personal (autoestima, confianza, creatividad) como desde una perspectiva general (creación de una atmósfera social agradable). En ese sentido, las posibilidades culturales que ofrecen los festivales, junto con la atmósfera de celebración que caracteriza a este tipo de eventos (Getz \& Cheyne, 1997; Waterman, 1998), pueden generar beneficios sociales que están relacionados, en términos generales, con la mejora del bienestar de los ciudadanos, la cohesión social y el desarrollo de valores cívicos. Todo ello como consecuencia de las oportunidades de aprendizaje de los eventos culturales, la exposición a la creatividad, la creación de redes y relaciones entre personas y la estrecha relación que los festivales tienen con el lugar en que se 
celebran (Moscardo, 2008). En definitiva, favorecen la creación de un sentido de lugar por parte de los ciudadanos, así como la generación de un espacio social y de interacción.

e) Repercusiones fisicas. Los festivales traspasan, en ocasiones, las repercusiones culturales y sociales y dejan una huella más profunda en el territorio, una huella física. La edificación de instalaciones fijas para el festival, la reordenación de espacios urbanos para su celebración, el desarrollo de actividades el resto del año o de nuevas zonas de la ciudad, pueden tener un reflejo en la arquitectura, el urbanismo y el uso de los espacios públicos y privados del lugar (Bianchini \& Parkinson, 1994; Evans, 2005; Hall, 2000). Este tipo de repercusiones son más patentes en el caso de los grandes eventos culturales, pero a una escala menor los festivales también impactan en el lugar donde se celebran.

En línea con estas ideas, el presente trabajo se centra fundamentalmente en el primero de los aspectos señalados anteriormente. Así, el objetivo fundamental de este artículo es determinar el impacto económico a corto plazo de un festival cultural sobre el territorio que lo acoge, por medio de la metodología de los Estudios de Impacto Económico, una aproximación basada en la definición y medición de tres tipos de efectos: los efectos directos, indirectos e inducidos. El prototipo de estudio es el Festival Internacional de Cine de Valdivia, uno de los festivales cinematográficos más importantes de Chile y un exponente interesante de un tipo de festival mediano, muy vinculado a la ciudad que lo acoge y con una evidente vocación cinéfila. Asimismo y como objetivo complementario, se analizan otras repercusiones que genera el festival desde los puntos de vista social, cultural o cinematográfico.

Partiendo de este planteamiento, el artículo se estructura en cinco apartados. Tras esta introducción, que ha pretendido reflejar la relación entre los festivales culturales y el territorio, se analiza y detalla la metodología de los Estudios de Impacto Económico, así como las particularidades de su aplicación al objeto de estudio. En el tercer apartado se presentan los resultados de la investigación, es decir, el impacto económico del festival. A continuación se analizan otras repercusiones de carácter social o cultural del evento. Finalmente, se desarrollan las principales conclusiones y reflexiones deducidas de la investigación.

\section{Metodología}

\section{Definición y metodología de los Estudios de Impacto Económico}

Los Estudios de Impacto Económico (EIE), también conocidos como método de los efectos, tratan de estimar la importancia económica de las artes y analizar los flujos de actividades e ingresos vinculados a la existencia de una determinada manifestación cultural (Martinello \& Minnon, 1990). Aunque su definición varía de unos casos a otros, el objetivo fundamental de estos estudios es medir los efectos derivados de la presencia de una actividad u organización cultural sobre una de- 
terminada área geográfica y en un determinado periodo. Se trata, en definitiva, de hacer aparecer los grandes flujos de rentas generados por la actividad cultural sobre la economía local o regional.

Estos estudios no son especialmente complicados desde el punto de vista conceptual, pero presentan algunas limitaciones y dificultades técnicas que han generado críticas, así como el planteamiento de metodologías alternativas de evaluación económica. El principal problema deriva del mal uso que se ha dado a veces a este tipo de estudios al tratar de justificar la intervención pública en cultura, ya que en los EIE no hay comparación entre alternativas de gasto posibles. Además, estos estudios no tienen en cuenta las repercusiones negativas que se pueden generar en el lugar (aumento del gasto en servicios de limpieza o seguridad, por ejemplo), que sí analizan otras metodologías. Finalmente, dificultades técnicas (conocimiento del número de espectadores, análisis depurado del gasto, modelización del tejido productivo, etcétera) y análisis poco cuidadosos han dado lugar a resultados exagerados en algunos estudios. Pero, a pesar de las limitaciones, el interés de los EIE deriva de la escrupulosidad en el método y de la realización del trabajo de campo para conocer los flujos económicos generados por la cultura, evitando sobredimensionamientos. En ese sentido, existen ejemplos interesantes y acreditados en el ámbito de los festivales culturales, entre los que podemos destacar los trabajos de Capaul (1988); Devesa Fernández (2006); The City of Edinburgh Council (2005); Herrero, Sanz, Bedate, Devesa y Del Barrio (2004); Perles Ribes, 2006; o Tyrrel e Ismail (2005).

Los EIE tienden a adoptar una metodología común, aunque existen diferencias entre unas aplicaciones y otras. En términos generales, distinguen tres tipos de efectos o impactos (Seaman, 2003). En primer lugar, los efectos directos, o gastos de la institución cultural analizados en términos de salarios, compras u otros conceptos. Son medidos mediante los presupuestos de la institución, es decir, por el análisis contable de los ingresos y gastos. En este sentido, se debe separar el gasto realizado en el área de referencia y el gasto realizado fuera de la misma, para poder asignarlo correctamente al ámbito de estudio. En segundo lugar tenemos los efectos indirectos, o gastos que realizan los espectadores como consecuencia del consumo cultural (entradas, alojamiento, comidas, etcétera). Suelen ser estimados por una encuesta al público asistente en la que se pregunta al encuestado por el gasto que espera realizar. Esta categoría presenta varias dificultades, que analizaremos en el siguiente apartado del artículo. Por último, los efectos inducidos son las repercusiones económicas de los gastos anteriores en el resto de la economía por medio del concepto multiplicador. Existen diferentes maneras de abordar el análisis del multiplicador, siendo habitual en los estudios de cultura y de turismo el uso de los multiplicadores insumo-producto derivados de las tablas del mismo nombre.

El análisis insumo-producto trata de medir los efectos que produce una variación en la demanda final sobre el sistema productivo en las Tablas Insumo-Producto (TIP) desarrolladas por Leontief en los años treinta. Las TIP son un instrumento económico que permite fotografiar la estructura económica de una zona y mostrar las interrelaciones entre todos los agentes de la economía (Cuadrado \& 
Arranz, 1996). Las TIP están formadas por tres grandes matrices — matriz de consumos intermedios, matriz de demanda final y matriz de inputs primarios-, siendo la más sustantiva la matriz de consumos intermedios, ya que en ella se desagrega la economía de un país o región en un determinado número de ramas de actividad (actividades homogéneas) y se muestran las relaciones de compraventa entre estas ramas o sectores (para un mayor detalle de la metodología Insumo-Producto puede consultarse Cuadrado \& Arranz, 1996; Muñoz Cidad, 2000; Pulido \& Fontela, 1993; Uriel, 1997).

Operando la matriz de consumos intermedios y definiendo los coeficientes técnicos, elemento fundamental del análisis de Leontief, llegamos a la matriz inversa de Leontief $(\mathrm{I}-\mathrm{A})^{-1}$, formada por elementos $\alpha_{\mathrm{ij}}$ que miden el esfuerzo productivo total que debe realizar la rama i si desea satisfacer un aumento unitario en la demanda final de los productos de la rama j. La suma de los elementos de cada columna son los multiplicadores sectoriales, que muestran cuánto tendrá que incrementarse la producción global del sistema productivo para satisfacer un aumento de una unidad en la demanda final de productos de la rama j. Es decir, en qué medida j difunde su propia actividad a lo largo del sistema productivo (Pulido \& Fontela, 1993).

\section{Aplicación de los Estudios de Impacto Económico al caso del Festival Interna- cional de Cine de Valdivia}

El Festival Internacional de Cine de Valdivia (FICV) nació en 1994 bajo iniciativa y amparo de la Universidad Austral de Chile y es considerado, hoy, uno de los festivales cinematográficos más importantes del país. Entre sus objetivos figuran contribuir al desarrollo de la industria audiovisual chilena, presentar nuevas propuestas cinematográficas y ser un lugar de encuentro de la industria audiovisual. A la vez, el festival establece como meta contribuir al desarrollo cultural de la nueva región de Los Ríos, ser una ventana de la región hacia el mundo y contribuir al turismo de la zona. En definitiva, ayudar al desarrollo y difusión de la industria audiovisual regional y nacional, contribuyendo así a la descentralización de la actividad cultural y económica del país. ${ }^{1}$

El FICV se celebra a lo largo de seis días del mes de octubre y está organizado en torno a cinco secciones fijas (muestra oficial) y una serie de secciones no competitivas dedicadas a diferentes autores, temas o cinematografías (muestra paralela). Organiza, asimismo, otras actividades para el público y los profesionales del sector, como foros, conferencias, talleres o la Mesa de Negocios, que constituye un espacio para la compraventa de películas. Un total de seis salas de cine de la ciudad acogen las proyecciones en una media de cinco sesiones diarias en diferentes horarios del día, que en 2008 fueron seguidas por casi 17.000 espectadores.

La aplicación de la metodología de los estudios de impacto al caso concreto del FICV exige, lógicamente, una serie de matices y adaptaciones a las particularidades del caso de estudio. Así, brevemente, debemos comentar esos matices, recordando

1 Página web del FICV: http://www.ficv.cl/ 
previamente que el objetivo fundamental del estudio es medir el impacto económico generado por este evento cinematográfico sobre la economía de la ciudad de Valdivia y la región de Los Ríos en 2008. De este modo, los efectos directos son los gastos realizados por el Festival Internacional de Cine de Valdivia en la zona de referencia y han sido obtenidos a partir de los presupuestos del festival de 2008. Los datos fueron proporcionados por la propia organización del festival, quien a su vez discriminó qué tipo de gastos eran ejecutados propiamente en la región y cuáles debían imputarse fuera de este espacio regional.

En cuanto a los efectos indirectos, estos son los gastos de los espectadores no locales o turistas relacionados con la celebración del festival, y fueron evaluados mediante una encuesta al público asistente. Esta categoría presenta varias dificultades, que señalamos a continuación, a la vez que explicamos cómo se han abordado en nuestro caso concreto de estudio:

a) Doble contabilización de ciertas partidas de gasto en los efectos directos e indirectos, que lleva a sobredimensionar los resultados. Este problema se ha solucionado no incluyendo, entre las categorías de gasto de la encuesta, las entradas y los productos que vende el festival (libros, carteles, etcétera), ya que están en la contabilidad del festival y, por tanto, en la categoría de impacto anterior.

b) Determinación de la entrada neta de dinero, es decir, el gasto de las personas que vienen de fuera o turistas. La determinación de la entrada neta de dinero en la economía local como consecuencia de la celebración del festival se realizó preguntando a los asistentes por su lugar habitual de residencia, lo que nos ha permitido distinguir entre el público local y el público no local. El gasto del público local no se incluye, en principio, en el estudio de impacto económico, ya que no es una entrada neta de dinero, sino una reasignación de la demanda; es decir, se trata de un gasto que no se perdería de no celebrarse el evento, sino que se realizaría en otras partidas y consumos. El gasto de los espectadores de fuera sí se tiene en cuenta, puesto que si no hubiese festival, se perdería definitivamente. Por lo tanto, el impacto indirecto está estrechamente vinculado a la capacidad de atracción de visitantes foráneos y, así, a la dimensión turística del festival.

c) Determinación de los gastos realmente imputables al festival, especialmente en el caso de los viajes con varios propósitos. El gasto realmente imputable al FICV se ha determinado con una pregunta a los asistentes no locales sobre el motivo de su viaje a Valdivia. Si el motivo del viaje es fundamentalmente el festival, se asigna el 100 por ciento del gasto señalado; si el festival es uno de los motivos de la presencia en la ciudad, se asigna el 50 por ciento; y si el festival no ha influido en su decisión de viaje, no se tiene en cuenta ningún gasto (véase Devesa Fernández, 2006; Herrero et al., 2004; Stanley, Rogers, Smeltzer \& Perron, 1998).

d) Finalmente, para asignar correctamente el gasto realizado en la economía de la ciudad y de la región se preguntó a los encuestados por el gasto medio diario 
realizado en la zona, siendo, por tanto, el propio espectador el encargado de discriminar entre el gasto total que esperaba realizar en el viaje y qué parte realizaría específicamente en Valdivia y la región de Los Ríos.

La encuesta al público asistente fue realizada durante la decimoquinta edición del FICV, celebrada entre el 3 y el 8 de octubre de 2008. El sistema de encuesta escogido fue el de "entrega-recogida" o encuesta autocumplimentada, por el cual se entregaba la encuesta a la entrada de la sesión cinematográfica y debía ser devuelta a la salida. El sistema de muestreo fue de tipo aleatorio, consistente en la entrega de una encuesta a espectadores de diferentes ciclos, salas, horarios y días de celebración del festival. Un total de 1.860 cuestionarios fueron entregados, de los cuales 750 fueron contestados y devueltos, lo que supone una tasa de respuesta del 40 por ciento. Las 750 encuestas válidas suponen un error muestral del $\pm 3,6$ por ciento para un nivel de confianza del 95 por ciento.

Finalmente y para cerrar el proceso metodológico, los efectos inducidos del modelo de impacto económico han sido calculados mediante los multiplicadores insumo-producto derivados de las Tablas Insumo-Producto de Chile para el año 2003, elaboradas por el Banco Central de Chile (2006), y que son las últimas disponibles para la realización de este estudio. En ese sentido, hemos clasificado los efectos directos e indirectos del modelo en cinco categorías diferentes de gasto y, para el cómputo de los efectos inducidos, hemos aplicado tres multiplicadores sectoriales (comercio, hoteles y restaurantes; transportes y comunicaciones; servicios empresariales) y un multiplicador general para el epígrafe de los sueldos y salarios. Este último se ha calculado como media ponderada de los multiplicadores sectoriales según el peso específico de cada sector en la economía.

\section{Resultados}

\section{El impacto del Festival Internacional de Cine de Valdivia}

Comenzando por el cómputo de los efectos directos, el FICV gastó en 2008 un total de 220,4 millones de pesos, de los que el 76,5 por ciento, es decir, 168,7 millones, quedó en la economía de la región (Cuadro 1). Es el gasto realizado en la región, proporcionado por la organización del festival, el que se tiene en cuenta en el estudio de impacto económico.

Del dinero gastado en la economía regional, la partida más importante es la relacionada con la programación ( 42,8 por ciento del total), en la que quedan reflejados fundamentalmente el alquiler de las salas de proyección, la publicidad, el transporte de las películas, la organización de la Mesa de Negocios o las publicaciones del festival. En segundo lugar, destaca la partida de sueldos y salarios del personal (27,3 por ciento), seguida del gasto en invitados (17,1 por ciento) y en la administración del evento (7,5 por ciento).

Para calcular, en segundo lugar, los efectos indirectos, debemos conocer específicamente varios datos, obtenidos en su mayoría en la encuesta realizada al público asistente: 
- Número de espectadores diferentes: cociente entre el número total de asistentes (15.698) y el número de películas vistas en media por los espectadores $(8,29)$; un total de 1.894 personas distintas.

- Origen de los espectadores, para distinguir entre los espectadores locales (48 por ciento del total, es decir, 909 espectadores distintos) y no locales (52 por ciento del total o 985 espectadores diferentes); solo el gasto de los turistas (espectadores no locales) entra en el estudio.

- Motivo de asistencia de los espectadores no locales, para poder asignar correctamente el gasto realizado: el 83,9 por ciento tuvo el festival como principal motivo de su viaje a Valdivia; 10,1 por ciento se desplazó a la ciudad por varios motivos, incluido el FICV; el 6,0 por ciento restante (cuyo gasto no entra en el estudio de impacto) declaró que su presencia en la ciudad no tenía nada que ver con el festival. En términos reales, esto se traduce en 826 espectadores distintos que tuvieron el festival como motivo principal del viaje, y 99 como motivo secundario.

- Gasto medio diario por partidas, segmentado por motivo del viaje.

- Número medio de días y noches de estancia en la zona, en función del motivo de asistencia. Los espectadores que tuvieron el festival como motivo principal asistieron una media de 4,4 días y una noche menos. Los espectadores que se desplazaron por varios motivos asistieron una media de 4,3 días y una noche menos.

Una vez obtenidos todos estos datos, podemos realizar los cálculos pertinentes para el cómputo de los efectos indirectos en el impacto económico del festival, tal y como se muestra en detalle en el Cuadro 2 y de manera resumida en el Cuadro 3.

\section{CUADRo 1 | Gasto del FICV (efecto directo)}

\begin{tabular}{|c|c|c|c|c|c|}
\hline \multirow{2}{*}{ PARTIDAS } & \multirow{2}{*}{$\begin{array}{l}\text { GASTO TOTAL } \\
\text { (PESOS) }\end{array}$} & \multirow{2}{*}{$\begin{array}{l}\text { GASTO FUERA } \\
\text { DE LA REGIÓN } \\
\text { (PESOS) }\end{array}$} & \multicolumn{2}{|c|}{ GASTO EN LA REGIÓN } & \multirow{2}{*}{$\begin{array}{l}\text { \% GASTO } \\
\text { REALIZADO } \\
\text { EN LA REGIÓN } \\
\text { SOBRE EL TOTAL }\end{array}$} \\
\hline & & & (PESOS) & US $\$^{A}$ & \\
\hline Personal & 53.856 .430 & 7.858 .519 & 45.997 .911 & 88.154 & 85,4 \\
\hline Invitados & 28.873 .735 & 0 & 28.873 .735 & 55.336 & 100,0 \\
\hline Programa & 72.774 .512 & 555.810 & 72.218 .702 & 138.406 & 99,2 \\
\hline Administración & 34.221 .577 & 21573123 & 12.648 .454 & 24.241 & 37,0 \\
\hline Premios & 22.763 .286 & 21.756 .936 & 1.006 .350 & 1.929 & 4,4 \\
\hline Otros & 8.000 .000 & 0 & 8.000 .000 & 15.332 & 100,0 \\
\hline TOTAL & 220.489 .540 & 51.744 .388 & 168.745 .152 & 323.397 & 76,5 \\
\hline
\end{tabular}

FUENTE ELABORACIÓN PROPIA SOBRE LA BASE DE DATOS APORTADOS POR LA ORGANIZACIÓN DEL FICV.

a'Tipo de Cambio promedio en 2008 Según el Banco Central de Chile: 521,79 pesos por dólar. 
CUADRO 2 | Gasto de los asistentes no locales al FICV

\begin{tabular}{|c|c|c|c|c|c|c|}
\hline & \multicolumn{3}{|c|}{ MOTIVO PRINCIPAL } & \multicolumn{3}{|c|}{ MOTIVO SECUNDARIO } \\
\hline & $\begin{array}{l}\text { GASTO MEDIO } \\
\text { DIARIO }^{A} \\
\text { (PESOS) }\end{array}$ & $\begin{array}{l}\text { ESTANCIA } \\
\text { MEDIA }^{A} \\
\text { (DÍAS/ } \\
\text { NOCHES) }\end{array}$ & $\begin{array}{l}\text { GASTO } \\
\text { ESTANCIA } \\
(100 \%)\end{array}$ & $\begin{array}{l}\text { GASTO MEDIO } \\
\text { DIARIO }^{A} \\
\text { (PESOS) }\end{array}$ & $\begin{array}{l}\text { ESTANCIA } \\
\text { MEDIA }^{A} \\
\text { (DÍAS/ } \\
\text { NOCHES) }\end{array}$ & $\begin{array}{l}\text { GASTO } \\
\text { ESTANCIA }^{\text {C }} \\
(50 \%)\end{array}$ \\
\hline Alojamiento & 4.722 & 3,4 & 13.105 .867 & 3.276 & 3,3 & 531.869 \\
\hline Comidas & 4.352 & 4,4 & 15.674 .772 & 3.690 & 4,3 & 781.690 \\
\hline Entretenimiento & 1.486 & 4,4 & 5.350 .592 & 1.509 & 4,3 & 319.616 \\
\hline Transportes & 739 & 4,4 & 2.660 .597 & 1.069 & 4,3 & 226.471 \\
\hline Compras & 1.844 & 4,4 & 6.641 .852 & 2.707 & 4,3 & 573.483 \\
\hline TOTAL & 13.143 & & 43.433 .680 & 12.250 & & 2.433 .130 \\
\hline
\end{tabular}

fuente elaboración propia sobre la base de la Encuesta al Público Asistente.

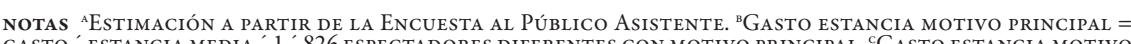
GASTO 'ESTANCIA MEDIA' 1 ' 826 ESPECTADORES DIFERENTES CON MOTIVO PRINCIPAL. 'GASTO ESTANCIA MOTIVO SECUNDARIO = GASTO 'ESTANCIA MEDIA ' $1 / 2$ ' 99 ESPECTADORES DIFERENTES CON MOTIVO SECUNDARIO.

CUADRO 3 | Gasto total del público asistente (efecto indirecto)

\begin{tabular}{|c|c|c|c|}
\hline & \multicolumn{2}{|c|}{ GASTO TOTAL } & \multirow{2}{*}{$\%$} \\
\hline & PESOS & US\$ $\$^{A}$ & \\
\hline Alojamiento & 13.637 .736 & 26.136 & 29,7 \\
\hline Comidas & 16.456 .462 & 31.538 & 35,9 \\
\hline Entretenimiento & 5.670 .208 & 10.867 & 12,4 \\
\hline Transportes & 2.887 .068 & 5.533 & 6,3 \\
\hline Compras & 7.215 .336 & 13.828 & 15,7 \\
\hline TOTAL & 45.866 .809 & 87.903 & 100,0 \\
\hline
\end{tabular}

fuente elaboración propia sobre la base de la Encuesta al Público Asistente.

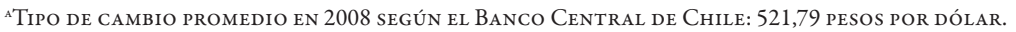

Como puede observarse, el efecto indirecto del FICV sobre la economía de la ciudad de Valdivia y la región de Los Ríos fue de 45.866 .809 pesos. De estos gastos, la parte más importante corresponde a la partida de comidas (35,9 por ciento del total), seguida del alojamiento (29,7 por ciento) y de las compras (casi el 16 por ciento). El gasto total de las personas que se desplazaron a Valdivia motivadas principalmente por el festival ascendió a 43,4 millones de pesos, y el gasto de los asistentes que señalaban tener varios motivos para estar en la ciudad durante el festival fue de 2,4 millones de pesos. 
Una vez conocidos los gastos directos e indirectos, podemos reorganizarlos en una serie de categorías básicas y calcular los efectos inducidos por medio de los multiplicadores interiores sectoriales de Chile para el año 2003, últimos existentes y los más apropiados para el estudio. ${ }^{2}$ Tal y como se ha señalado anteriormente, se han aplicado tres multiplicadores sectoriales y uno general (sueldos y salarios), que es una media ponderada de todos los multiplicadores sectoriales en función del peso de la producción de cada sector. Los multiplicadores utilizados corresponden a la TIP de doce sectores de la economía chilena. ${ }^{3}$ Los resultados aparecen recogidos en el Cuadro 4.

CUADRO 4 | Efectos inducidos sobre la economía regional

\begin{tabular}{|c|c|c|c|c|c|c|}
\hline & $\begin{array}{l}\text { GASTOS } \\
\text { DIRECTOS } \\
\text { (A) }\end{array}$ & $\begin{array}{c}\text { GASTOS } \\
\text { INDIRECTOS } \\
\text { (в) }\end{array}$ & $\begin{array}{l}\text { SUMA DE } \\
\text { GASTOS } \\
(\mathrm{C}=\mathrm{A}+\mathrm{B})\end{array}$ & $\begin{array}{c}\text { MULTIPLICADOR: } \\
\text { SECTORES } \\
\text { UTILIZADOS }^{A}\end{array}$ & $\begin{array}{c}\text { MULTIPLICADOR }^{A} \\
\text { (D) }\end{array}$ & $\begin{array}{l}\text { EFECTO } \\
\text { INDUCIDO } \\
\left(\begin{array}{l}\text { C X D })\end{array}\right.\end{array}$ \\
\hline $\begin{array}{l}\text { Personal y } \\
\text { premios }\end{array}$ & 47.004 .261 & - & 47.004 .261 & M General & 1,6503 & 77.569 .285 \\
\hline $\begin{array}{l}\text { Alojamiento, } \\
\text { restauración y } \\
\text { entretenimiento }\end{array}$ & 36.873 .735 & 35.764 .406 & 72.638 .141 & $\begin{array}{l}7 \text { Comercio, hoteles } \\
\text { y restaurantes }\end{array}$ & 1,7796 & 129.269 .750 \\
\hline Compras & 7.250 .272 & 7.215 .336 & 14.465 .608 & $\begin{array}{l}7 \text { Comercio, hoteles } \\
\text { y restaurantes }\end{array}$ & 1,7796 & 25.743 .575 \\
\hline $\begin{array}{l}\text { Transportes y } \\
\text { comunicaciones }\end{array}$ & 18.871 .058 & 2.887 .068 & 21.758 .126 & $\begin{array}{l}8 \text { Transporte y } \\
\text { comunicaciones }\end{array}$ & 1,6197 & 35.242 .440 \\
\hline $\begin{array}{l}\text { Servicios a } \\
\text { empresas }\end{array}$ & 58.745 .826 & - & 58.745 .826 & $\begin{array}{l}9 \text { Intermediación } \\
\text { financiera y servicios } \\
\text { empresariales }\end{array}$ & 1,4494 & 85.143 .742 \\
\hline TOTAL & 168.745 .152 & 45.866 .809 & 214.611 .961 & & & 352.968 .792 \\
\hline
\end{tabular}

FUENTE ELABORACIÓN PROPIA.

aBanco Central de Chile (2006).

La repercusión o gasto total del festival sobre la economía de la ciudad es la suma de los efectos anteriormente descritos: un total de 352 millones de pesos que se diluyen por el tejido empresarial de la zona de referencia, tal y como queda reflejado en el Cuadro 5.

2 En el caso de los gastos directos, la agrupación se ha realizado gracias al detalle en las partidas de gasto del presupuesto del festival proporcionado por la organización, que ha permitido clasificar el gasto según las categorías establecidas en el Cuadro 4, para poder aplicar los multiplicadores insumo-producto. En el caso de los gastos indirectos, hemos agrupado las categorías de alojamiento, comidas y entretenimiento del Cuadro 3 en una sola partida, y conservado el detalle de las otras dos (véase Cuadro 3).

3 En realidad, los multiplicadores aplicados hacen referencia a la economía de Chile, y no de la región de Los Ríos, ya que no existen Tablas Insumo-Producto para la región. Existe, por tanto, un desfase en los efectos inducidos que supone uno de los límites de la investigación y que nos hace considerar los efectos inducidos y, por tanto, el efecto total, como un máximo, aunque los multiplicadores regionales suelen ser menores que los multiplicadores nacionales. 
CUADRO 5 | Impacto total del FICV

\begin{tabular}{l|c|c}
\hline & PESOS & US\$ ${ }^{\mathrm{A}}$ \\
\hline Gasto directo & 168.745 .152 & 323.397 \\
Gasto indirecto & 45.866 .809 & 87.903 \\
Gasto inducido neto & 138.356 .830 & 265.158 \\
\hline EFECTO TOTAL & 352.968 .792 & 676.458 \\
\hline
\end{tabular}

FUENTE ELABORACIÓN PROPIA.

aTipo de Cambio promedio en 2008 Según el Banco Central de Chile: 521,79 pesos por dólar.

Los sectores más beneficiados por la celebración del festival son hostelería y restaurantes, es decir, el sector turístico de la ciudad (Figura 1). En segundo lugar, los servicios a las empresas, ya que un festival es una actividad que reclama numerosos servicios empresariales. Los transportes y comunicaciones son el tercer sector más beneficiado (10 por ciento de los efectos), y el sector comercial recibe el 7,3 por ciento de los beneficios del festival, ya que ingresan, en una semana, más de 25 millones de pesos. Finalmente, 22 por ciento no puede ser asignado directamente a ningún sector de actividad, ya que deriva de las contribuciones monetarias en sueldos y salarios, por lo que su detalle exigirá un estudio posterior.

FIGURA 1 | Distribución del efecto total por sectores (porcentajes)

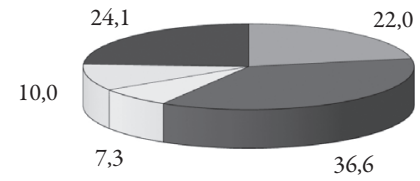

Personal y premios

Alojamiento, restauración y entretenimiento

$\square$ Compras

$\square \quad$ Transportes y comunicaciones

- Servicios a empresas

FUENTE ELABORACIÓN PROPIA. 
Los efectos que acabamos de analizar hacen referencia al impacto económico del FICV a corto plazo sobre la ciudad y región donde se celebra. En este caso solo hemos tenido en cuenta, en los efectos indirectos, el gasto de los espectadores de fuera de la zona, es decir, el gasto de los visitantes, que es el caso más extremo y ortodoxo desde el punto de vista económico. Sin embargo, en algunas ocasiones es posible tener en cuenta también el gasto de los espectadores locales, normalmente en el caso de eventos culturales que, por su carácter extraordinario, generan en los ciudadanos unos gastos de renta adicionales y fuera del rango ordinario.

En el caso del FICV, el 42,5 por ciento de los espectadores locales señala que su asistencia al festival le genera un desembolso económico superior al habitual. Así, si tenemos en cuenta el gasto en comidas, entretenimiento, transporte y compras de esos espectadores locales, quienes de media asisten 3,4 días al evento, podríamos añadir 6.875 .847 pesos adicionales a los efectos indirectos. Aplicando los correspondientes multiplicadores insumo-producto sectoriales, esa cifra se eleva a 11.996 .271 pesos. En definitiva, si añadimos el gasto de los espectadores locales - basándonos en una cierta excepcionalidad del festival que conlleva un mayor consumo de los espectadores de la zona-, el impacto económico total del festival en la ciudad de Valdivia y la región de Los Ríos sería de 364.965 .063 pesos (699.448 dólares estadounidenses).

\section{El impacto del festival en contexto}

En definitiva, la celebración del Festival Internacional de Cine de Valdivia genera diversas rentas que se dispersan por la economía de la ciudad y de la región y que contribuyen al tejido productivo de la zona, al empleo de la ciudad y al desarrollo del sector audiovisual y cultural de Valdivia. Lógicamente, el impacto es mayor en el ámbito geográfico más cercano al epicentro de la actividad cultural, la ciudad de Valdivia, diluyéndose a medida que nos alejamos del mismo, es decir, en el resto de la región de Los Ríos.

No es fácil poner en relación el impacto económico generado por el FICV con la economía de la zona, como consecuencia de la falta de datos sobre la estructura económica regional y local. ${ }^{4}$ La región de Los Ríos supone el 4,5 por ciento del territorio chileno, el 2,5 por ciento de la población y, aproximadamente, el 1,3 por ciento del PIB del país (Ministerio de Obras Públicas [MOP], 2010a). Los sectores económicos más destacados, aun cuando no existe detalle sobre la estructura productiva de la región, son la actividad agropecuaria-silvícola (destacando especialmente el sector de la madera), la industria manufacturera y los transportes y comunicaciones (MOP, 2010a). Como podemos observar, no destaca el sector servicios, aunque todo apunta a que en la provincia y en la comuna de Valdivia los servicios y el turismo tienen un papel mayor que en el conjunto de la región, debido a la propia entidad demográfica de Valdivia y la importancia de algunas de sus actividades más características (turismo, universidad, conocimiento, etcétera).

4 La región de Los Ríos, XIV Región, fue creada formalmente el 15 de marzo de 2007. No existen todavía datos sobre el PIB regional ni sobre su estructura por sectores de actividad, que estarán disponibles, según el Instituto Nacional de Estadística de Chile —organismo encargado de su elaboración— a partir de 2012. 
Según un estudio del año 2000 sobre la estructura productiva de la provincia de Valdivia (Coper, Mery \& Retamal, 2000), el capital de la provincia se concentraba, en 1999, en la industria manufacturera (31 por ciento), el sector comercio, restaurantes y hoteles ( 24 por ciento) y el sector de servicios financieros, seguros y profesionales (15 por ciento), destacando dentro de la primera las industrias alimentaria y de la madera. Esta estructura productiva también se da en la comuna, aunque el peso del sector servicios es mayor que en el conjunto de la provincia. Por ello, la iniciativa del Festival Internacional de Cine de Valdivia ayuda a desarrollar el tejido productivo y empresarial de la ciudad y contribuye a uno de los sectores económicos más potentes de la zona, el sector turístico y comercial, en un proceso generalizado de terciarización de las economías y de crecimiento de la economía cultural, creativa y del ocio.

Además, el festival encaja muy bien en algunos de los retos que se plantean para la región de Los Ríos y para la ciudad de Valdivia en los próximos años. En el plan "Región de Los Ríos 2020. Obras públicas para el desarrollo” (MOP, 2010b) se establecen como objetivos estratégicos, entre otros, posicionar la región como factor de identidad regional y nacional, por su naturaleza, creatividad y multiculturalidad; desarrollar un turismo de intereses especiales de naturaleza y patrimonio histórico —en el que el festival puede ser complementario a esa oferta-; impulsar el desarrollo de las industrias del conocimiento y creativa y cultural, lo que apunta a potenciar, entre otras cosas, el fortalecimiento del polo audiovisual del sur, vinculado a su vez con el festival.

En esa misma línea, la ciudad de Valdivia puso en marcha en 2008 el proyecto "Valdivia: Ciudad universitaria y del conocimiento", que tiene como principal objetivo potenciar la ciudad como un gran centro universitario, científico-tecnológico y del conocimiento en el sur de Chile. ${ }^{5}$ Aunque la idea surgió para crear un gran cluster universitario y del conocimiento, pretende también mejorar y desarrollar la actividad cultural y artística de la zona y, así, la calidad de vida de sus ciudadanos. En definitiva, Valdivia está tratando de desarrollar una nueva economía, basada en el conocimiento, la cultura, la creación, el turismo o el cine, en la que el FICV puede, y de hecho ya es así, contribuir.

\section{Otras repercusiones del festival}

Las repercusiones económicas no son los únicos efectos del festival sobre el territorio. Tal y como hemos señalado anteriormente, los festivales tienen resonancias culturales y sociales sobre el lugar en que se organizan y que, en esencia, son la verdadera razón de ser de los mismos.

Así, aun cuando no tenemos indicadores específicos ni se han desarrollado estudios concretos para el caso de estudio, conocemos la opinión de los espectadores sobre dichas repercusiones, lo que nos permite una aproximación a este importante

Véase, en ese sentido, el Protocolo de Acuerdo "Valdivia: Ciudad universitaria y del conocimiento", firmado el 22 de septiembre de 2008 por la Municipalidad de Valdivia y las universidades y centros de estudios científicos de la región (http://www.munivaldivia.cl/ciudad_universitaria/protocolo_de_acuerdo.htm). 
aspecto del festival. En la encuesta realizada al público asistente los espectadores tenían que valorar en una escala de 1 a 7 (siendo 1 nada y 7 mucho) la importancia que ellos creían que el festival tenía sobre diferentes aspectos. Los resultados aparecen recogidos en la Figura 2.

\section{FIGURA 2 | Valoración de las repercusiones del festival}

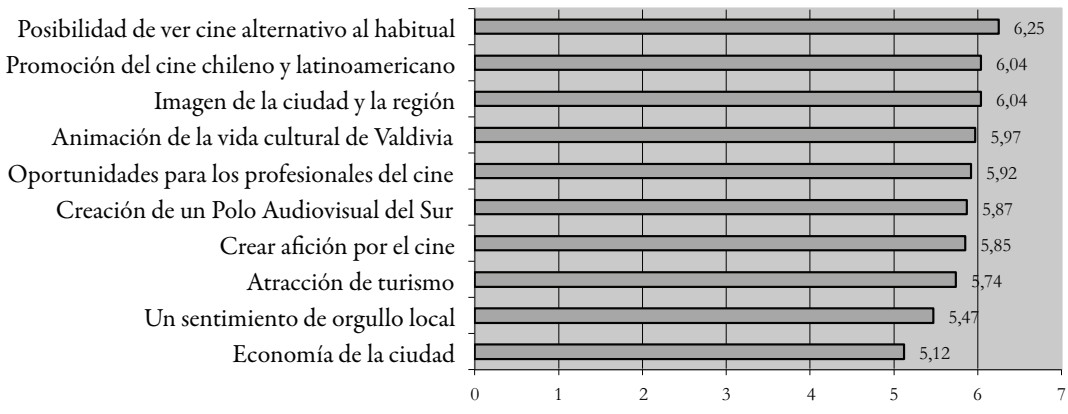

La valoración que los espectadores hacen de todos los aspectos propuestos es, en general, muy elevada, aunque algunas repercusiones son, a juicio de los asistentes, más importantes que otras. Así, la valoración más alta es la posibilidad que ofrece el festival de ver un cine diferente al que habitualmente se encuentra en las salas cinematográficas; un aspecto importante, ya que uno de los principales objetivos de este tipo de festivales es, precisamente, el de ampliar la oferta cinematográfica y presentar películas de calidad que no encuentran otros canales de distribución. En segundo lugar, y muy relacionado con el anterior, destaca la posibilidad de promoción para el cine chileno y latinoamericano, reconociéndose así el papel de los festivales como circuitos de distribución y exhibición cinematográfica y, por tanto, su papel en la industria audiovisual. Los espectadores también valoran muy positivamente el impacto que el festival tiene sobre la imagen de la zona y la animación cultural de la ciudad, poniéndose en evidencia sus beneficios para los habitantes del lugar.

Se reconocen igualmente otros efectos del festival sobre el sector cinematográfico, como las oportunidades para los profesionales de la industria, la posibilidad de crear en la ciudad un Polo Audiovisual del Sur y de generar una cierta afición por el cine.

Menos valoradas por los participantes del festival serían sus repercusiones sobre la atracción de turismo y la creación o aumento de un cierto sentimiento de orgullo local. El impacto menos valorado, aun cuando es de 5,12 sobre 7, sería el económico, es decir, la capacidad del FICV de generar producción, rentas y empleos. Y ello a pesar de que el estudio realizado pone de manifiesto que el festival genera un impacto económico que para nada puede considerarse como despreciable, sino que, por el contrario, resulta bastante significativo.

En definitiva, los espectadores reconocen especialmente las repercusiones culturales del festival en un doble sentido: los beneficios culturales para ellos como 
consumidores de cine (posibilidad de ver cine alternativo) y como ciudadanos (animación de la ciudad); y los beneficios culturales para el sector (profesionales, empresas, etcétera). También son conscientes de la capacidad del festival de mejorar la imagen de la ciudad, aun cuando su apreciación del impacto del festival sobre el turismo, la creación de un sentido de lugar y la economía sea relativamente menor.

Estos datos no revelan específicamente las opiniones y sentimientos de los ciudadanos de Valdivia y, por tanto, el papel del festival en el bienestar de la sociedad local, porque lo que se ha efectuado es un estudio global de la valoración de todos los asistentes al festival. Sin embargo, el análisis de las diferencias en la valoración de las repercusiones del FICV entre espectadores locales (de la ciudad y la región) y no locales (de fuera de la región), nos permite avanzar algunas ideas. Los espectadores locales valoran en general más que los espectadores no locales todas las repercusiones del festival, destacando especialmente tres: la posibilidad de ver cine diferente, la animación de la ciudad y la imagen de la zona. ${ }^{6}$ Son tres impactos que afectan no solo a las personas que asisten al festival, sino al conjunto de la ciudadanía, confirmando de alguna manera los beneficios sociales de este tipo de eventos.

Por otra parte, un indicador indirecto del bienestar y aceptación generado por el festival es el hecho de que en el 94,2 por ciento los espectadores de 2008 señalaron que tenían intención de repetir su asistencia al siguiente año, y 97,2 por ciento recomendaría el FICV a familiares y amigos. El festival parece cumplir, en ese sentido, sus objetivos culturales y sociales.

\section{Conclusiones}

En los últimos años, la cultura ha pasado a formar parte de las estrategias de desarrollo local y regional y de las actuaciones de regeneración urbana, no solo por su potencial para generar riqueza y empleo, sino por su contribución a la diversificación del producto turístico, su capacidad para impulsar la cohesión social y el bienestar de los ciudadanos o su aporte a la diferenciación de los lugares. Los festivales culturales, una de las manifestaciones artísticas más dinámicas e interesantes del panorama cultural actual, también forman parte de las políticas de desarrollo económico, como consecuencia de las múltiples repercusiones - económicas, sociales, culturales, turísticas o físicas- que generan en los territorios donde se celebran.

En este artículo hemos analizado el impacto económico del Festival Internacional de Cine de Valdivia en la ciudad de Valdivia y la región de Los Ríos, por medio de la metodología de los Estudios de Impacto Económico. Estos estudios tratan de medir los efectos económicos a corto plazo de un evento cultural por la definición y evaluación de tres tipos de flujos o efectos: los efectos directos, los efectos indirectos y los efectos inducidos, o efectos de arrastre de los dos anteriores sobre el tejido productivo local. Así, el Festival Internacional de Cine de Valdivia generó en 2008 ingresos en la economía regional que se cifraron en 352,9 millones de pesos, en su

6 Las diferencias en la valoración de los dos primeros aspectos (cine diferente y animación de la ciudad) son significativas desde un punto de vista estadístico, aunque no en el caso de la valoración de la imagen de la zona. 
sentido más estricto y ortodoxo, pues solo se han considerado entradas asociadas a los asistentes no locales y se ha discriminado entre los que tuvieron un motivo principal en la asistencia al evento cultural. El festival afecta especialmente al sector turístico de la ciudad, así como a los servicios a las empresas. Genera, en definitiva, una circulación activa de fondos entre los agentes de la economía local y regional, tanto públicos como privados, así como un gasto superior de los espectadores locales que impacta también sobre el territorio.

El estudio pone de manifiesto, en ese sentido, la importancia del turismo como canal de transmisión fundamental y más evidente del impacto económico a corto plazo de las actividades culturales —en este caso, del festival — sobre la economía local y regional. También parece demostrarse que los efectos económicos territoriales de este tipo de eventos son de radio corto, en el sentido de que el impacto se produce de forma fundamental en la ciudad que acoge el evento y se va diluyendo según nos alejamos geográficamente del núcleo fundamental de gasto. Por consiguiente, debemos señalar que el estudio - y la metodología de los Estudios de Impacto Económico, en términos generales - no evalúa si las acciones culturales son en lo social rentables en el largo plazo, ni si son consistentes con un desarrollo económico y cultural sostenible y vinculado a aspectos como la mejora de la imagen, del nivel educativo, del bienestar de la sociedad, o cambios más profundos en el tejido productivo y en el sector cultural de la zona.

Sin embargo, aun cuando no tenemos indicadores concretos en ese sentido - ya que el objetivo fundamental del estudio es la estimación del impacto económico a corto plazo del festival de cine-, sí conocemos las apreciaciones y opiniones de los espectadores del festival sobre algunos de los impactos sociales o culturales del evento. Los aspectos mejor valorados son sus repercusiones culturales, tanto desde un punto de vista específico (posibilidad de ver cine alternativo, oportunidades para los profesionales y para el sector cinematográfico en la zona) como desde un punto de vista general (animación del lugar). Menos importancia relativa dan los asistentes a los posibles beneficios en la atracción de turismo, mejoras sociales o impacto económico.

Estas apreciaciones, lejos de ser irrelevantes, aciertan en la función fundamental de los festivales culturales en general y cinematográficos en particular. Los festivales de cine se han convertido, hoy, en un canal importantísimo para la distribución y exhibición cinematográfica, especialmente para las películas más pequeñas, independientes o vanguardistas, que no encuentran fácilmente el camino de llegada a las salas cinematográficas y, cuando tienen calidad suficiente, utilizan el circuito de festivales para iniciar su carrera comercial. Pero también los festivales de cine se han convertido en un lugar fundamental para el consumo cinematográfico, debido a que permiten ver un cine diferente al de las salas comerciales e incluso, en las localidades más pequeñas, constituyen casi el único lugar donde ver cine en una pantalla grande.

En definitiva, los festivales tienen un papel fundamental a la hora de diversificar la oferta cultural de las ciudades y regiones, preservar la cultura de la sociedad, mejorar el bienestar de los ciudadanos y aumentar la cohesión social. Deben contribuir, así, al desarrollo económico y al progreso social y cultural de los territorios. @EURE 


\section{Referencias bibliográficas}

Banco Central de Chile (2006). Cuentas nacionales de Chile. Compilación de referencia 2003. Santiago: Autor. En http://www.bcentral.cl/publicaciones/estadisticas/actividad-economicagasto/aeg15.htm

Bianchini, F. \& Parkinson, M. (Eds.). (1994). Cultural policy and urban regeneration. The West European experience. Manchester: Manchester University Press.

Capaul, M. (1988). El impacto económico del Festival Internacional de Cine de San Sebastián. Estudios Empresariales, 67, 48-54.

Chacko, H. \& Schaffer, J. (1993). The evolution of a festival. Tourism Management, 14(6), 475-482. En http://dx.doi.org/10.1016/0261-5177(93)90100-Y

Coper, S., Mery, C. \& Retamal, J. M. A. (2000). Informe sobre la estructura económica de la provincia de Valdivia. [mimeo].

Crespi-Vallbona, M. \& Richards, G. (2007). The meaning of cultural festivals. Stakeholder perspectives in Catalunya. International Journal of Cultural Policy, 13(1), 103-122. doi http:// dx.doi.org/10.1080/10286630701201830

Crompton, J. L. \& McKay, S. L. (1997). Motives of visitors attending festival events. Annals of Tourism Research, 24(2), 425-439. En http://dx.doi.org/10.1016/S0160-7383(97)80010-2

Cuadrado, J. R. \& Arranz, A. (1996). Los impactos económicos del turismo desde la perspectiva del análisis input-output. En A. Pedreño \& V. Monfort (Eds.), Introducción a la economía del turismo en España (pp. 181-216). Madrid: Civitas.

Devesa Fernández, M. (2006). El impacto económico de los festivales culturales. El caso de la Semana Internacional de Cine de Valladolid. Madrid: Fundación Autor.

Evans, G. (2001). Cultural planning: an urban renaissance. Londres: Routledge.

Evans, G. (2005). Measure for measure: Evaluating the evidence of culture's contribution to regeneration. Urban Studies, 42(5/6), 959-983. doi: 10.1080/00420980500107102

Florida, R. L. (2002). The rise of the creative class: And how it's transforming work, leisure, community and everyday life. New York: Basic Books.

Frey, B. \& Busenhart, I (1996). Special exhibitions and festivals: Culture's booming path to glory. En V.A. Ginsburg \& M. Menger (Eds.), Economics of the Arts. Selected Essays (pp. 275-302). Ámsterdam: Elsevier Science.

Frey, B. S. (1994). The economics of music festivals. Journal of Cultural Economics, 18(1), 29-39. doi: $10.1007 / \mathrm{BF} 01207151$

Getz, D. \& Cheyne, J. (1997). Special event motives and behaviour. En C. Ryan (Ed.), The Tourist Experience (pp. 136-154). Londres: Cassell.

Getz, D. (1997). Event management \& event tourism. Nueva York: Cognizant Communication Corporation.

Gibson, L. \& Stevenson, D. (2004). Urban space and the uses of culture. International Journal of Cultural Policy: 10(1), 1-4. doi: 10.1080/1028663042000212292

Grant, D. \& Paliwoda, S. (1998). Segmenting Alberta Arts and Festival Consumers. Festival Management and Event Tourism, 5(4), 207-220.

Hall, P. (2000). Creative cities and economic development. Urban Studies, 37(4), 639-649. doi: $10.1080 / 00420980050003946$ 
Herrero Prieto, L. C. (2011). La contribución de la cultura y las artes al desarrollo económico regional. Investigaciones Regionales, 19, 177-202. En http://redalyc.uaemex.mx/src/inicio/ ArtPdfRed.jsp?iCve $=28918214010$

Herrero, L. C., Sanz, J. A., Bedate, A., Devesa, M. \& Del Barrio, M. J. (2004). Turismo cultural e impacto económico de Salamanca 2002, Ciudad europea de la cultura. Madrid: Civitas.

Lim, H. (1993). Cultural strategies for revitalizing the city: A review and evaluation. Regional Studies, 27(6), 589-594.

Martinello, M. \& Minnon, M. (1990). Les études d'impact: objetifs et méthodes. En R. Wangermee (Ed.), Les malheurs d'Orphee. Culture et profit dans l'économie de la musique (pp. 127-141). Bruselas: Pierre Mardaga Editeur.

Ministerio de Obras Públicas (MOP), Chile. (2010a). Chile 2020. Obras públicas para el desarrollo. Santiago: Autor. En http://www.dirplan.cl/planes/vision2020/Documents/Chile2020. pdf. Versión digital en www.mop.cl/2020

Ministerio de Obras Públicas (MOP), Chile. (2010b). Región de Los Ríos 2020. Obras públicas para el desarrollo [extracto del libro Chile 2020. Obras públicaspara el desarrollo]. Santiago: Autor. En http://www.dirplan.cl/planes/regionales/Documents/XIV/MOP\%20SEPARATA\%20LOS\%20RIOS\%2018-12.pdf.

Moscardo, G. (2008). Analyzing the role of festivals and events in regional development. Event Management, 11(1-2), 23-32.

Muñoz Cidad, C. (2000). Las cuentas de la Nación. Introducción a la Economía Aplicada. Madrid: Civitas.

Perles Ribes, J. F. (2006). Análisis del impacto económico de eventos: una aplicación a fiestas populares de proyección turística. Cuadernos de Turismo, 17, 147-166. En http://redalyc.uaemex. $\mathrm{mx} / \mathrm{src} /$ inicio/ArtPdfRed.jsp?iCve=39801707

Pulido, A. \& Fontela, E. (1993). Análisis input-output. Modelos, datos y aplicaciones. Madrid: Pirámide.

Quinn, B. (2005). Arts festivals and the city. Urban Studies, 42(5/6), 927-943. doi: $10.1080 / 00420980500107250$

Richards, G. \& Palmer, R. (2010). Eventful cities. Cultural management and urban revitalisation. Oxford: Elsevier.

Richards, G. \& Wilson,J. (2004). The impact of cultural events on city image: Rotterdam, cultural capital of Europe 2001. Urban Studies, 41(10), 1931-1951. doi: 10.1080/0042098042000256323

Richards, G. (2007). The festivalization of society or the socialization of festivals? The case of Catalunya. En G. Richards (Ed.), Cultural tourism: Global and local perspectives (pp. 257-279). Binghampton: Haworth Hospitality Press.

Scott, A. J. (2000). The cultural economy of cities. London: Sage.

Seaman, B. (2003). Economic impact of the arts. En R. Towse (Ed.), A handbook of Cultural Economics (pp. 224-231). Cheltenham: Edward Elgar Publishing.

Stanley, D., Rogers, J., Smeltzer, S. \& Perron, L. (1998). Win, place or show: Gauging the economic success of the Renoir and Barnes art exhibits. Quebec: Department of Canadian Heritage.

The City of Edinburgh Council (2005). Edinburgh's year round festivals 2004-2005 economic impact study [mimeo]. Disponible: http://efaextra.efa-aef.eu/efadoc/11\%5Cfestivals_ exec_summary_final_\%20edinburgh\%2004-05.pdf 
Tyrrel, B. J. \& Ismail, J. A. (2005). A methodology for estimating the attendance and economic impact of an open-gate festival. Event Management, 9(3), 111-118.

Uriel, E. (1997). Contabilidad nacional. Barcelona: Ariel.

Waterman, S. (1998). Carnivals for elites? The cultural politics of arts festivals. Progress in Human Geography, 22(1), 54-74. doi: 10.1191/030913298672233886

Weiler, B. \& Hall, C. M. (1992). Special interest tourism. Londres: Belhaven Press. 